\title{
Calidad de vida en niños con leucemia linfoblástica aguda en el hospital pediátrico de Sinaloa mediante el PedsQL cancer module
}

\section{Quality of life in children with acute lymphoblastic leukemia in the pediatric hospital of Sinaloa through the PedsQL cancer module}

\author{
Amparo Guadalupe Rodríguez-Alvarado ${ }^{1 *}$, Saúl Canizales-Muñoz², Mirna Ríos-Osuna ${ }^{3}$, Alberto Páez-Sala- \\ $\operatorname{zar}^{4}$
}

1. Especialidad en Neonatología. Hospital Pediátrico de Sinaloa, "Dr. Rigoberto Aguilar Pico", Culiacán Sinaloa, México.

2. Departamento de Investigación, Hospital Pediátrico de Sinaloa, "Dr. Rigoberto Aguilar Pico", Culiacán Sinaloa, México

3. Servicio de Hematología y Oncología, Hospital General Regional \# 1 Instituto Mexicano del Seguro Social. Culiacán Sinaloa, México.

4. Jefe de Enseñanza Médica. Hospital Pediátrico de Sinaloa, “Dr. Rigoberto Aguilar Pico”, Culiacán Sinaloa, México.

Hospital Pediátrico de Sinaloa "Dr. Rigoberto Aguilar Pico", Culiacán, Sinaloa.

Teléfonos (667)713-90-04 extensión 291. Dirección: Blvd. Constitución S/N, Colonia Jorge Almada, CP 80200,

Culiacán, Sinaloa, México.

*Autor de correspondencia: Amparo Guadalupe Rodríguez-Alvarado

Correo electrónico: rodriguez_roal@hotmail.com Teléfono Celular: 3121358617 / 3234981

DOI http://dx.doi.org/10.28960/revmeduas.2007-8013.v12.n1.004

Recibido 10 de agosto 2021, aceptado 12 de octubre 2021

RESUMEN

Objetivo: evaluar y analizar la calidad de vida de los pacientes pediátricos diagnosticados con leucemia linfoblástica aguda en pacientes de 2 a 18 años mediante el instrumento PedsQL 3.0 cancer module. Material y métodos: Se aplicó el instrumento PedsQL cancer module a 44 pacientes con leucemia linfoblástica aguda y sus cuidadores del Hospital Pediátrico de Sinaloa "Dr. Rigoberto Aguilar Pico" con el fin de analizar su confiabilidad y validez, determinar las dimensiones más afectadas de acuerdo a los ítems evaluados por el instrumento así como comparar las puntuaciones de la calidad (CV) de vida de acuerdo con la edad, género y etapa de tratamiento. Resultados: Se verificó la confiabilidad y validez del instrumento para medir la calidad de vida. Los ítems más afectados fue la preocupación, seguido por la ansiedad a los procedimientos; el ítem con mayor puntuación fue la comunicación. Los pacientes con mejor calidad de vida fueron los que se encontraban en vigilancia, seguido de los que se encontraban en recaída y tratamiento. Conclusiones: El PedsQL cancer module es un instrumento confiable y válido para evaluar la calidad de vida en los pacientes, el conocimiento del mismo ayudará al tratamiento integral de estos pacientes y a la elaboración de estrategias que mejoren sus condiciones.

Palabras clave: Calidad de vida, leucemia linfoblástica aguda, cáncer, PedsQL cancer module.

\section{ABSTRACT}

Objective: To evaluate and analyze the quality of life of pediatric patients diagnosed with acute lymphoblastic leukemia in patients aged 2 to 18 years using the PedsQL 3.0 cancer module instrument. Material and methods: The PedsQL cancer module instrument was applied to 44 patients with acute lymphoblastic leukemia and their caregivers at the "Dr. Rigoberto Aguilar Pico "in order to analyze its reliability and validity, determine the most affected dimensions according to the items evaluated by the instrument, as well as compare the quality of life scores according to age, gender and stage of treatment. Results: The reliability and validity of the instrument to measure quality of life was verified. The items most affected were concern, followed by anxiety about procedures; the item with the highest score was communication. The patients with the best quality of life were those who were under surveillance, followed by those who were in relapse and treatment. Conclusions: The PedsQL cancer module is a reliable and valid instrument to assess the quality of life in patients, knowledge of it will help the comprehensive treatment of these patients and the development of strategies to improve their conditions.

Keywords: Quality of life, acute lymphoblastic leukemia, cancer, PedsQL cancer module

\section{Introducción}

La leucemia linfoblástica aguda (LLA) es la neo-

plasia maligna más frecuente en los niños y re-

presenta $25 \%$ de todos los tipos de cáncer en

éstos ${ }^{5}$. Cada año, más de 160,000 menores de
20 años son diagnosticados con cáncer en países desarrollados ${ }^{7}$. La incidencia de cáncer infantil es generalmente entre $100-180$ por 1 000,000 de niños al año ${ }^{1}$. 
México no es la excepción y aproximadamente cada año 7,000 niños adquieren la enferme$\mathrm{dad}^{7}$. Ocupa el séptimo lugar en cuanto a mortalidad en niños de uno a cuatro años de edad, con una tasa de 3.2/100 000 habitantes y en los niños de 5 a 14 años es la segunda causa de muerte, sólo después de los accidentes de tránsito de vehículo de motor, con una tasa de 2.7/ 100000 habitantes $^{5}$.

En el pasado, un diagnóstico de la leucemia linfoblástica aguda significaba una cierta fatalidad. Sin embargo, en las últimas cinco décadas, las tasas de supervivencia de la leucemia infantil han aumentado, especialmente en los últimos 40 años, en los que el avance significativo en los protocolos terapéuticos ha permitido reducir las tasas de mortalidad. Debido a ello, muchos pacientes pediátricos de cáncer pueden curarse si son diagnosticados y tratados tempranamente $^{2}$. Con los tratamientos disponibles en la actualidad, más de $80 \%$ de los niños con leucemia linfoblástica aguda pueden sobrevivir ${ }^{5}$.

Aunque la tasa de supervivencia es una variable de resultados relativamente fáciles de evaluar para determinar el pronóstico de los pacientes con cáncer, no refleja apropiadamente el impacto que tiene sobre cada niño, particularmente ante las experiencias subjetivas, como su estado de salud ${ }^{2}$. La calidad de vida relacionada con la salud está influenciada por la modalidad de tratamiento y la fase de la terapia ${ }^{1}$. Además las decisiones sobre la implementación de mejoras en la asistencia sanitaria pública pueden adoptarse sobre la base del impacto de las intervenciones sobre la calidad de vida ${ }^{2}$, la cual se define como un constructo multidimensional que integra la percepción del paciente ante el impacto de la enfermedad y su tratamiento, así como su funcionamiento en diversos aspectos de la vida, incluyendo las áreas física, psicológica y de la salud social ${ }^{2}$. La información sobre la calidad de vida de los pacientes de cáncer pediátrico y sus familias permite identificar a las familias con necesidades especiales de apoyo o intervención psicológica².

La evaluación de CV inició con la limitante de basarse en medicines blandas, con la característica de ser subjetivas, por lo que requirió métodos de evaluación válidos, reproducibles y confiables. Por ello, entre más se describan las evaluaciones para medir CV mejorará la incorporación de estos instrumentos en la evaluación integral de individuos, en la conducción de los ensayos clínicos y en la investigación de servicios de salud ${ }^{5}$.

La Organización Mundial de la Salud define la calidad de vida relacionada con la salud como la percepción individual de su posición en la vida, en el contexto de la cultura y los sistemas de valores, es multidimensional y generalmente incluye dominios psicológicos, físicos y sociales. En los niños, el funcionamiento cognitivo, la autonomía, la imagen corporal y las relaciones 
familiares pueden ser incluidos para una evaluación confiable de la calidad de vida relacionada con la salud'.

La evolución del estudio de la calidad de vida ha permitido que los instrumentos que la evalúan sean más precisos. En la actualidad se dispone de escalas o instrumentos que pueden ser genéricos o específicos ${ }^{2}$.

EI PedsQLTM (por sus siglas en inglés, Pediatric Quality of Life Inventory) es una serie instrumentos genéricos se han utilizado para medir calidad de vida a pacientes sanos y enfermos en edades de 2 a 18 años con enfermedades agudas y crónicas ${ }^{2}$. Están constituido por cuestionarios que se aplican tanto para pacientes como para sus cuidadores, el cual permite seleccionar una de las 5 opciones de la escala Likert que le asigna una puntuación. El total de los valores más altos indica mejor salud en relación a calidad de vida ${ }^{11}$.

En pacientes pediátricos oncológicos se ha utilizado el instrumento el PedsQL 3.0 cancer module para medir el impacto del cáncer en la calidad de vida de los niños y adolescentes. Este instrumento se desarrolló específicamente. Para medir el impacto de los síntomas y el tratamiento en la calidad de vida de los pacientes con cáncer. Este instrumento tiene la ventaja de ser multidimensional, específico para cáncer, de fácil comprensión y diseño para pacientes pediátricos entre las edades de 2 a 18 años.
Además, está disponible en versión a manera de autorreporte diseñada para niños/adolescentes y de manera paralela para que cuidadores evalúen la calidad de vida1.

El objetivo de este artículo es evaluar y analizar la calidad de vida de los pacientes pediátricos diagnosticados con leucemia linfoblástica aguda en pacientes de 2 a 18 años mediante el instrumento PedsQL 3.0 cancer module.

\section{Material y métodos}

Se realizó un estudio observacional, descriptivo, transversal y prospectivo. La muestra se conformó por 44 pacientes de 2 a 18 años que acudieron a la consulta de urgencias, consulta de hemato-oncología y hospitalización del Hospital Pediátrico de Sinaloa "Dr. Rigoberto Aguilar Pico" durante los meses de marzo de 2019 a septiembre de 2019. Los pacientes se incluyeron de forma consecutiva, no aleatoria. Se excluyeron niños con padecimientos neurológicos o cognitivos agregados o con alguna otra enfermedad crónica que impidiera la realización del cuestionario.

Previo a la aplicación del instrumento se obtuvieron datos de los pacientes como: nombre, edad, sexo, fecha del diagnóstico y etapa del tratamiento (tratamiento activo, recaída o vigilancia) así como en sus cuidadores se obtuvo nombre, edad y parentesco. 
Se aplicó el instrumento PedsQL cancer module a 44 pacientes con diagnóstico de Leucemia Linfoblástica Aguda así como a sus acompañantes, previa autorización por el familiar y por el comité de ética del Hospital, dividiéndose en cuatro grupos de edad, de acuerdo a los cuestionarios: de 2-4, 5-7, 8-12 y de 13-18 años. El cuestionario se aplicó por el investigador de manera estandarizada, quienes no sabían leer o escribir se les leyeron las preguntas y se marcó el cuestionario por el entrevistador. Se excluyeron 6 pacientes así como sus cuidadores, uno por presentar patología neurológica y los 5 restantes presentaron los cuestionarios incompletos.

Este instrumento tiene diferentes dimensiones que evalúan varios aspectos de $\mathrm{CV}$ y tiene versiones distintas de acuerdo a los grupos de edad ya predeterminados. A las respuestas se le asigna un puntaje con cinco opciones, variando de un puntaje 0 (nunca ha sido un problema) hasta 4 (casi siempre ha sido un problema). Para obtener el puntaje global, cada una de las respuestas se codifica a escalas lineales inversas de 0-100, siendo 100 la calificación más alta y mejor. A cada valor se le asigna un puntaje de la siguiente forma: $0=100,1=$ $75,2=50,3=25$ y $4=0$. El puntaje total es el resultado de la suma de la escala lineal. Este instrumento también es multidimensional y evalúa ocho dominios: dolor y molestias, presencia de náusea, ansiedad por procedimientos, ansie- dad por tratamientos, preocupaciones, problemas cognitivos, percepción de apariencia física y comunicación.

Se contactó con el autor intelectual del instrumento (J.W. Varni) ${ }^{7}$ quien autorizó la utilización del cuestionario en sus versiones traducidas al español con fines académicos.

\section{Resultados}

Se registraron un total de 50 pacientes y sus padres o tutores de marzo a septiembre de 2019 , se los cuales se excluyeron 5 pacientes y sus padres por no obtener datos completos, así como 1 paciente y su familiar por presentar padecimiento neurológico que impidió realizar la encuesta. De los 44 pacientes restantes se les aplicó al $100 \%$ el instrumento PedQLs cancer module versión 3.0.

Nuestro estudio se realizó en pacientes con diagnóstico de LLA en edad de 2 a 18 años, encuestando a un total de 44 pacientes, la edad más frecuente de los pacientes fue 7 años con un porcentaje de $13.3 \%$, el $46.7 \%$ de los pacientes tenía más de 10 años, la mayor edad fue 18 años y la menor 3 años con una diferencia de 15 años. Predominó el sexo masculino en un $75.6 \%$ de los casos con una relación hombre: mujer 3:1. Se reportaron un total de 34 pacientes del sexo masculino y 11 del sexo femenino con un porcentaje de $24.4 \%$ (Cuadro 1). 
Cuadro 1. Característica demográfica y clínicas de los pacientes con leucemia linfoblástica aguda.

\begin{tabular}{lc}
\hline Paciente & $\mathrm{n}(\%)$ \\
\hline Edad (años) & $4(9.1)$ \\
$2-4$ & $13(29.5)$ \\
$5-7$ & $13(29.5)$ \\
$8-12$ & $14(31.8)$ \\
$13-18$ & \\
& \\
Género & $10(22.7)$ \\
Femenino & $34(77.3)$ \\
Masculino & \\
Etapa de tratamiento & \\
Inducción a remisión & $1(2.3)$ \\
Mantenimiento & $24(54.5)$ \\
Vigilancia & $11(25)$ \\
Consolidación & $1(2.3)$ \\
Recaída & $7(15.9)$ \\
\hline
\end{tabular}

En las fases del tratamiento el $48.9 \%$ se encontraba en mantenimiento, debido a que esta fase de tratamiento dura de 2 a 3 años, por lo tanto, es mayor porcentaje de pacientes que están en mantenimiento. Del resto de los pacientes, $2.2 \%$ se encontraba en inducción a la remisión, $4.4 \%$ en mantenimiento posterior a 6 meses de recaída, $2.2 \%$ en consolidación, $24.4 \%$ en vigilancia, $6.7 \%$ en recaída, $2.2 \%$ en recaída inducción a la remisión, $2.2 \%$ se encontraba en su segunda recaída, $2.2 \%$ en su tercera recaída y $2.2 \%$ en su cuarta recaída.
En cuanto a los promedios y correlación de los resultados de PedsQL cancer module de pacientes y cuidadores y/o padres, de manera global y de sus ocho dimensiones se reportó una media en la dimensión del dolor de 69.85 del paciente y 73.52 del cuidador, en náusea se reportó una media de 80.52 del paciente y 81.92 del cuidador, en la ansiedad por los procedimientos se reportó una media de 34.95 por el paciente y 33.54 por el cuidador, en la preocupación se reportó una media de 18.51 por el paciente y 11.28 por el cuidador, en cuanto a los problemas cognitivos se reportó una media de 78.88 por el paciente y 75.74 por parte del cuidador, en la apariencia física se reportó una media de 83.75 por el paciente y 86.87 por el cuidador y en la comunicación se reportó una media de 96.58 por parte del paciente y 96.58 por parte del cuidador. Los resultados totales de la prueba y de cada dimensión para los pacientes y los cuidadores en general, el promedio de las calificaciones obtenidas entre pacientes y cuidadores son muy similares corroborándose por el coeficiente de correlación intraclase. Asimismo, conviene mencionar que los dos dominios más afectados fueron preocupación con 18.51 y 11.28 y ansiedad por procedimientos con 34.95 y 33.54 respectivamente. La comunicación fue la mejor puntuación que se obtuvo. (Cuadro 2). 
Cuadro 2. Comparación de la percepción de la calidad de vida de los pacientes y sus cuidadores en relación a la salud.

\begin{tabular}{llll}
\hline & $\begin{array}{l}\text { Pacientes } \\
(\mathrm{n}=44)\end{array}$ & $\begin{array}{l}\text { Cuidadores } \\
(\mathrm{n}=44)\end{array}$ & $\begin{array}{l}\text { Correlación intraclase } \\
(\mathrm{IC} 95 \%)\end{array}$ \\
\hline Dolor & 69.85 & 73.52 & $0.79(0.663-0.872)$ \\
Náuseas & 80.51 & 81.92 & $0.77(0.708-0.183)$ \\
Ansiedad por procedimientos & 34.95 & 33.54 & $0.82(0.75-0.87)$ \\
Ansiedad por tratamientos & 82.39 & 77.80 & $0.76(0.65-0.83)$ \\
Preocupación & 18.51 & 11.28 & $0.71(0.58-0.80)$ \\
Problemas cognitivos & 78.88 & 75.74 & $0.73(0.68-0.82)$ \\
Apariencia física & 83.75 & 86.87 & $0.82(0.74-0.87)$ \\
Comunicación & 96.58 & 96.58 & $0.86(0.80-0.90)$ \\
\hline
\end{tabular}

Para los pacientes y los cuidadores se comparó la calidad de vida en función de la edad de los pacientes; se observa cómo los pacientes de 11 a 14 años son quienes tienen las calificaciones más bajas, considerando la evaluación de los propios pacientes así como de sus cuidadores (con una media de 64.84 y 61.75 respectivamente. Los pacientes de 15 a 18 años obtuvieron las más altas calificaciones así como sus cuidadores con una media de 75.51 y 75.95 respectivamente.

Con respecto a la etapa del tratamiento, también hubo ciertas diferencias entre las calificaciones de pacientes y padres. Se reportó mejor calidad de vida en los pacientes que se encuentran actualmente en vigilancia así como los resultados obtenidos por los familiares con una media de 77.74 y 74.79 respectivamente. Sin embargo, desde el punto de vista estadístico esta diferencia entre los grupos no fue significativa. En cuanto a los pacientes que se encuentran en tratamiento y recaída se reportaron con baja calidad de vida reportándose una media de
65.5 y 67.24 respectivamente y de sus cuidadores en 64.77 y 67.32 respectivamente.

Finalmente, cuando se compararon los promedios de calidad con respecto al tiempo de evolución de la enfermedad a partir del diagnóstico Se reportó más baja calidad de vida durante el inicio del tratamiento a los 42 meses con una media de 65.57, mientras que la más alta calidad de vida se reportó dentro de los 86 a 128 meses de tratamiento con una media de 78.01.

\section{Discusión}

Nuestro objetivo primario fue evaluar la calidad de vida en los pacientes de 2 a 18 años con diagnóstico de Leucemia Linfoblástica Aguda del Hospital Pediátrico de Sinaloa y por tratarse de pacientes que cuentan con amplios recursos para el tratamiento de su padecimiento, su calidad de vida debía ser buena, así mismo nuestra intención es que con los resultados obtenidos podamos elaborar propuestas para mejorar los 
ámbitos en los que los resultados no hayan sido lo que esperábamos. En este estudio se aplicó la versión 3.0 del instrumento PedsQL cancer module de acuerdo a las edades entre 2 a 18 años.

Se analizó en el estudio de Dorantes-Acosta y cols $^{3}$ la Confiabilidad y validez de las respuestas entre padres e hijos, tras la aplicación del instrumento PCQL-3 en pacientes mexicanos reportándose adecuada con alfa de Cronbach en 0.834 , al igual que en nuestro estudio ya que se reporta en 0.77 .

Al comparar la CV en función del tiempo de conocer el diagnóstico (intervalos de 42 meses o menos, de 43 a 85 meses, de 83 a 128 y 129 o más), Ramírez-Zamora LM y colaboradores ${ }^{2}$ no encontraron diferencias significativas; sin embargo en nuestro estudio se observó un incremento de la calidad de vida acorde al tiempo diagnóstico, iniciando con una media de 65.57 a los 0 a 42 meses aumentando a 74.53 posterior a los 129 meses, los resultados de la prueba indican que en los pacientes hay una tendencia a mejorar su percepción de calidad de vida conforme transcurre el tiempo del diagnóstico. Lo anterior respalda lo observado en otras investigaciones que sustentan que los pacientes con mayor tiempo de conocer su diagnóstico presentan mejor puntuación en la escala de calidad de vida ${ }^{1}$.
De acuerdo a Álvarez-Hernández $\mathrm{G}$ y cols ${ }^{2}$ se apreciaron diferencias en la percepción de la calidad de vida entre los niños y sus tutores, calificando de mejor modo la calidad de vida que los niños, sin embargo en nuestro estudio se observó concordancia en la percepción de la calidad de vida de los pacientes y sus cuidadores. Al analizar la CV en función de la etapa de la enfermedad Ramírez-Zamora LM y colaboradores $^{2}$ obtuvieron mayor puntuación en los que se encontraban en cuidados paliativos, seguido por pacientes en vigilancia, tratamiento y recaída, comparado a nuestro estudio no contamos con área establecida de cuidados paliativos por lo cual no se realizó tal comparación, sin embargo se observó mayor puntuación en los que se encontraban en vigilancia con 77.74 y con la menor puntuación los que se encontraban en tratamiento con un $65.5^{2}$.

Con respecto a la comparación de los resultados de la CV en cuanto a la percepción de los padres o cuidadores, con la de los pacientes, se determinó una buena concordancia en todos los dominios del instrumento. Dorantes-Acosta refirieron buena concordancia al igual que el estudio realizado por Ramírez-Zamora LM y colaboradores $^{2}$ pero en el estudio de Villarruel y LucioGómez $z^{1}$ no se encontró concordancia. 


\section{Conclusiones}

El PedsQL cancer module es un instrumento confiable y válido para la evaluación de la CV en los pacientes mexicanos con cáncer, tanto para la versión para pacientes como sus cuidadores de 2 a 18 años. La ventaja de ser un instrumento de rápida y sencilla aplicación ayuda al profesional de la salud a detectar las condiciones que disminuyan la CV así como posterior a su identificación implementar estrategias específicas para brindar acciones e intervenciones para mejorar la CV del paciente y su entorno.

\section{Referencias}

1. Castillo-Martínez ID, Juárez-Villegas LE, Palomo-Colli MÁ, Medina-Sansón A, ZapataTarrés M. Calidad de vida en niños con leucemia linfoblástica aguda durante la inducción a la remisión mediante el PedsQL Cancer Module@ $\odot$. Boletín Médico del Hospital Infantil de México. 2009;66(5):410-8.

2. Varni JW, Burwinkle TM, Katz ER, Meeske $\mathrm{K}$, Dickinson $\mathrm{P}$. The PedsQL ${ }^{\mathrm{TM}}$ in pediatric cancer: reliability and validity of the pediatric quality of life inventory ${ }^{\mathrm{TM}}$ generic core scales, multidimensional fatigue scale, and cancer module. Cancer. 2002;94(7):2090-106.

3. Scarpelli AC, Paiva SM, Pordeus IA, Varni JW, Viegas CM, Allison PJ. The Pediatric Quality of Life Inventory ${ }^{\mathrm{TM}}$ (PedsQL ${ }^{\mathrm{TM}}$ ) family impact module: reliability and validity of the Brazilian version. Health and Quality of Life Outcomes. 2008;6(1):35.
4. Upton P, Eiser C, Cheung I, Hutchings HA, Jenney M, Maddocks A, et al. Measurement properties of the UK-English version of the Pediatric Quality of Life Inventory 4.0 (PedsQL) generic core scales. Health and quality of life outcomes. 2005;3:22-.

5. Álvarez-Hernández G, Reynoso-Angüis PA, Hurtado-Valenzuela JG, del Carmen Candia-Plata M, Pérez-Moya G. Calidad de vida de los niños con padecimientos crónicos en el Hospital Infantil de Sonora. Rev Mex Pediatr. 2014;81(2):48-55.

6. Spieth LE, Harris CV. Assessment of healthrelated quality of life in children and adolescents: an integrative review. Journal of pediatric psychology. 1996;21(2):175-93.

7. Fajardo-Gutiérrez A, Juárez-Ocaña $S$, González-Miranda G, Palma-Padilla V, CarreónCruz R, Ortega-Alvárez MC, et al. Incidence of cancer in children residing in ten jurisdictions of the Mexican Republic: importance of the Cancer registry (a population-based study). BMC cancer. 2007;7(1):68.

8. Velarde-Jurado E, Avila-Figueroa CJSpdM. Consideraciones metodológicas para evaluar la calidad de vida. 2002;44(5):448-63.

9. Memik NÇ, Ağaoğlu B, Coşkun A, Karakaya IJTJCAMH. The validity and reliability of pediatric quality of life inventory in 8-12 year old Turkish children. 2008;15(2):87-98.

10. Varni JW, Seid M, Kurtin PSJMc. PedsQL ${ }^{\mathrm{TM}}$ 4.0: Reliability and validity of the Pediatric Quality of Life Inventory ${ }^{\mathrm{TM}}$ Version $4.0 \mathrm{Ge}-$ neric Core Scales in healthy and patient populations. 2001:800-12. 
11. Varni JW, Seid M, Rode CAJMc. The PedsQL ${ }^{\mathrm{TM}}$ : measurement model for the pediatric quality of life inventory. 1999:126-39.

12. Dorantes-Acosta E, Villasís-Keever MÁ, Zapata-Tarrés M, Arias-Gómez J, EscamillaNúñez A, Miranda-Lora AL, et al. Calidad de vida de niños mexicanos con leucemia linfoblástica aguda atendidos por el Seguro Popular. Boletín médico del Hospital Infantil de México. 2012;69(3):242-54.
13. Ramírez-Zamora LM, Llamas-Peregriana N, Lona-Reyes JC, Sánchez-Zubieta FA. Calidad de vida en niños con cáncer mediante PedsQL Cancer Module@). Rev Mex Pediatr. 2015;82(2):49-56.

14. Rivas MBV, Gómez-Maqueo MELJPyS. Propiedades psicométricas del Inventario de Calidad de Vida Pediátrico, Versión 4.0, en adolescentes mexicanos. Psicología y Salud. 2010;20(1):5-12. 